\title{
Symmetries and symmetry-breakings: the fabric of physical interactions and the flow of time ${ }^{1}$
}

\author{
Giuseppe Longo \\ Dépt. d'Informatique, CNRS - Ecole Normale Supérieure \\ et CREA, Polytechnique, Paris \\ http://www.di.ens.fr/users/longo
}

\begin{abstract}
Summary. This short note develops some ideas along the lines of the stimulating paper by Heylighen (Found Sci 15 4(3):345-356, 2010a). It summarizes a theme in several writings with Francis Bailly, downloadable from this author's web page. The "geometrization" of time and causality is the common ground of the analysis hinted here and in Heylighen's paper. Heylighen adds a logical notion, consistency, in order to understand a possible origin of the selective process that may have originated this organization of natural phenomena. We will join our perspectives by hinting to some gnoseological complexes, common to mathematics and physics, which may shed light on the issues raised by Heylighen. Note: Francis Bailly passed away recently: his immense experience in physics has been leading our joint work for many years.
\end{abstract}

Historically, it is with relativist physics that there occurs a "change of perspective": we pass from "causal laws" to the structural organization of space and time, or even from causal laws to the "legality/normativity of geometric structures". This understanding of causal laws by the identification of structural organizations, stems essentially from the intrinsic duality existing between the characterization of the geometry of the universe and that of energymomentum within that universe. By this duality and the putting into effect of the principle of invariance under the differentiable transformations of space-time, the "forces" are relativized to the nature of this geometry: they will even appear or disappear according to the geometric nature of the universe chosen a priori to describe physical behaviors.

Now, it is similar for quantum physics, in gauge theories. Here, gauge groups operate upon internal variables, such as in the case of relativity, where the choice of local gauges and their changes enable to define, or conversely, to make disappear, the interactions characterizing the reciprocal effects of fields upon one another. For example, it is the choice of the Lorentz gauge, which enables to produce the potential for electromagnetic interactions as correlates to gauge invariance.

Consequently, if one considers that one of the modalities of expression and observation of the causal processes is to be found in the precise characterization of the forces and fields "causing" the phenomena observed, then it is apparent that this modality is profoundly thrown into question by the effects of these transformations. Not that the causal structure itself will as a result be intrinsically subverted, but the description of its effects is profoundly relativized.

This type of observation therefore leads to having a more elaborate representation of causality than that resulting from the first intuition stemming from classical behaviors. Particularly, the causality of contemporary physics seems much more associated to the manifestation of a formal solidarity of the phenomena between themselves, as well as between the phenomena and the referential frameworks chosen to describe them, than to an object's "action" oriented towards another in inert space-time, as classical mechanics could have accredited the idea. In summary, our strong stand towards a geometrization of causality may be summarized as follows. Causes become interactions and these interactions themselves constitute the fabric of the universe of their manifestations, its geometry: modifying this fabric appears to cause the interactions to change; changing the interactions modifies the fabric.

\footnotetext{
${ }^{1}$ In Foundations of Science, Vol. 16, Issue 4, pp. 331-333, 2011.
} 
And now comes another fundamental issue raised by Heylighen. It appears that the symmetry / symmetry breaking pair is playing for the intelligibility of physics an absolutely crucial role. By Noether's theorems, to which Heylighen refers, transformations in symmetry correspond invariants (mathematical aspect) or conserved quantities (physical aspect) specific to the system under consideration and to any systems displaying identical symmetries.

Thus, the symmetry / breaking of symmetry pair thematizes, on the one hand, invariance, conservation, regularity and equivalence, and on the other, criticality, instability, singularity, ordering. We have seen in the book quoted below that through the pair's dialectic, it is an essential component of the very identity of the scientific object that is presented and objectivized. Could we go even further and consider that we have thus managed construct this identity at a level such that cognitive schemas conceived as conditions of possibility for any construction of objectivity are henceforth mobilized, thus reviving a form of transcendental approach, in a kantian sense?

As a matter of fact, there exists a close formal relationship between the abstract properties of symmetry captured by mathematical group structures and logical structures as fundamental as the equivalence relation, which is extensively used by Heylighen. At the same time, there exists a similar formal relationship between the semi-group structure and the logical structure of the (partial) order relation, to which Heylighen refers in his understanding of time and causality. Thus, the theoretical analysis of the abstract notions of space and of time demonstrates that for their formal reconstruction these notions need to mobilize the mathematical structures of group and of semi-group, respectively. Indeed, regardless of the number of dimensions considered, the displacement properties, consubstantial to the concept of space, refer to the determinations of the displacement group, whereas the properties of irreversibility and of the passing of time refer to the characteristics of the semi-group (generally, for one parameter).

We then witness the constitution of a pair of abstract complexes which doubtlessly represents one of the essential bases for any objective interpretation within the processes of the construction of knowledge: the complexes of <space, group structure, equivalence relation $>$ on the one hand and of <time, semi-group structure, order relation on the other $>$. Epistemologically, this is where Heylighen's thesis leads, in our opinion. By adding "consistency" in the logical sense, Heylighen suggests a way to break circularities in the cyclic structure of equivalence relations and pass by this to order relations, that is to semigroup and time ("going back" to a node A from which one "moved away" is a form of opposite movement, a negation $\neg \mathrm{A}$, thus incompatible or logically inconsistent - one cannot have both $\mathrm{A}$ and $\neg \mathrm{A}$ ).

Let's point out once more that the space and time evoked by the gnoseological complexes above no longer refer to physical entities as such, but rather to the conceptual frameworks which are meant to enable any physics to manifest itself, that is, to abstract conditions of possibility and not to effective realizations, thus reactualizing a kantian point of view. Thus, space and time are no longer considered as "objects" to be studied, but rather as the conditions of possibility for any sensible experience. In this sense, the symmetries and breakings of symmetries associated to these complexes appear not only as elements of the intelligibility of physical reality, but indeed as factors for the scientific constitution of such reality, including the understanding of causality.

Not only would we simply operationalize space and time (and thus causality), but by coupling them with the corresponding logical and mathematical determinations (group structure, equivalence relation, etc.) we refer them to the frameworks of invariance which make them into reference structures that are mathematically specified, rather than abstract and vague. 


\section{References}

Bailly F., Longo G. (2006) Mathématiques et sciences de la nature. La singularité physique du vivant, Hermann, Paris (in English, Imperial College Press/ World Sci., 2011).

Heylighen, F. (2010a). The self-organization of time and causality: Steps towards understandingthe ultimate origin. Foundations of Science, 15, 4(3), 345-356. doi:10.1007/ s10699- 010-9171- 1.91 arXiv:0912.5508v2.92

Heylighen, F. (2010b). Symmetry, potentiality and reversibility. Foundations of Science. arXiv:0912.5508v2.

Longo G., Montévil M. (2011). From Physics to Biology by Extending Criticality and Symmetry Breakings. To appear in a special issue of Progress in Biophysics and Molecular Biology. 